\section{Ethnoepidemiology of postnatal depression}

\author{
Prospective multivariate study of sociocultural risk factors \\ in a Chinese population in Hong Kong
}

\author{
DOMINIC T. S. LEE, ALEXANDER S. K. YIP, TONY Y. S. LEUNG \\ and TONY K. H. CHUNG
}

\begin{abstract}
Background Although there have been many studies of the biological and psychosocial causations of postnatal depression, studies of sociocultural risk factors are rare.
\end{abstract}

Aims To investigate the sociocultural risk factors of postnatal depression using ethnographically informed epidemiological methods.

Method A total of 959 women were assessed at their first ante-partum visit (baseline), in the third trimester, immediately after delivery, and 3 months post-partum. Six domains of risk factors were examined. The dependent variable was postnatal depression (as defined by the Edinburgh Postnatal Depression Scale) at 3 months post-partum.

Results Conflict with mother-in-law, marital dissatisfaction, past depression and antenatal depression independently predicted the occurrence of postnatal depression. The cultural practice of peiyue - a Chinese post-partum custom of mandated family support - was associated with better social support and a slightly lower risk of postnatal depression.

Conclusions Sociocultural aspects of the immediate puerperium shape maternal emotional well-being. In-law conflict is an important source of household distress in many Asian societies. The findings have implications for clinical practice and future studies.

Declaration of interest None. Funding detailed in Acknowledgements.
In many industrialised countries postnatal depression has become the most common complication of childbirth. Epidemiological studies generally show that $10-15 \%$ of recently delivered women are affected by postnatal depression (Appleby et al, 1996; Brugha et al, 1998; Lee et al, 2001; Chandran et al, 2002). It is now recognised that the formation of postnatal depression is shaped by risk factors at several levels (O’Hara \& Swain, 1996). Marital discord, inadequate social support, past depression, personality vulnerability and life events during pregnancy or near delivery have consistently been shown to predict postnatal depression. A close connection between antenatal depressive mood and postnatal depression has also been observed. Sociocultural factors have also been implicated in the formation of postnatal depression (Cox, 1996). Inasmuch as postnatal depression is reportedly rare in preindustrialised societies (Becker, 1998), it has been suggested that postnatal depression is a culture-bound syndrome of Western modernity (Stern \& Kruckman, 1983). However, empirical studies of the sociocultural aetiology of postnatal depression have been few and far between.

\section{METHOD}

\section{Participants}

To investigate the sociocultural risk factors of postnatal depression using an epidemiological method that is ethnographically informed, 959 participants were recruited among consecutive women who attended the antenatal booking clinic of the Prince of Wales Hospital in Hong Kong. The hospital is a university-affiliated public hospital that serves a million people of diverse socio-economic background. Women were excluded from the study only if they were not Chinese in ethnicity, did not have long-term residential rights, were leaving Hong Kong within 12 months of delivery, or did not supply informed written consent. The research protocol was approved by the university's clinical research ethics committee.

\section{Study design}

After a complete description of the study to the participants, their written informed consent was obtained. We recruited the participants at the first antenatal assessment (baseline) and reassessed them in their third trimester (30-32 weeks of gestation), immediately after delivery and at 3 months post-partum. At baseline, the research nurses collected socio-demographic, medical, obstetric and psychiatric data from the participants in a semi-structured manner. Putative risk factors of postnatal depression were examined at baseline as far as possible. Risk factors that could not be assessed at baseline were measured in the earliest feasible assessment.

\section{Putative risk factors}

We examined the risk factors that are commonly reported in previous studies of postnatal depression. We also assessed ethnocultural risk factors of local importance. Six domains of risk factors were included:

(a) demographic and socio-economic factors: age, marital status, number of children, recent immigration, education, unemployment, being a 'housewife' ('home-maker'), household income, financial difficulty and social class;

(b) medical and psychiatric history: past depressive episode, past deliberate selfharm, history of prolonged (more than 4 weeks) hypnotic use, family psychiatric history, previous miscarriage, previous induced abortion, past infertility and history of major medical illnesses;

(c) ante-partum depressive symptoms: level of depression at 30-32 weeks of gestation;

(d) perinatal stressor: emergency Caesarean delivery, failed breast-feeding and neonatal admission;

(e) interpersonal relationships: marital dissatisfaction, poor social support and partner non-participation in baby care;

(f) sociocultural factors: peiyue support practice (being accompanied and assisted by a designated female relative 
or friend for more than $4 \mathrm{~h}$ per day in the first month), exemption from house chores as a result of peiyue, mother-in-law relationship, small accommodation $\left(<28 \mathrm{~m}^{2}\right)$ and dissatisfaction with accommodation.

A number of sociocultural risk factors have been proposed for postnatal depression. One focus of attention has been the dissolution of traditional cultural rites and values in modern motherhood. In preindustrial societies the post-partum period is patterned by elaborate customs and rituals, many of which mobilise social support and call attention to maternal vulnerability. In modern culture, such customs and rituals rarely exist (Pillsbury, 1978; Stern \& Kruckman, 1983; Cox, 1996).

A commonly cited example of socioprotective post-partum custom is mandated family support. In most contemporary Asian societies, recently delivered women are still regarded as vulnerable, and are exempted from their usual household duties for an extended period (Pillsbury, 1978; Grace et al, 2001). Such exemption is generally made possible by the continual presence of a designated elder female kin, most commonly the woman's mother or mother-in-law. This practice, called peiyue in the Chinese culture, consolidates and formalises post-partum support, guarantees passage of child-rearing knowledge, and protects the mother from exhaustion and sleep deprivation. Social anthropologists and cultural psychiatrists have long suggested that peiyue, as a socio-moral custom that 'mothers the mother', may prevent social isolation and postnatal depression (Pillsbury, 1978). On the other hand, these post-partum support practices bring in extended family members, such as in-laws, who may not be welcomed by the new mothers. For centuries, tense relationships between women and their mothers-in-law have been a source of household disharmony in Chinese and many other Asian societies (Chen, 1999). A qualitative phenomenological study conducted by our group found that the enriched social contact ritualised by peiyue is not necessarily perceived as supportive (Chan et al, 2002). There were also reports of mother-in-law problems in postnatal depression of other cultures (Barrows $\&$ Barrows, 2002; Chandran et al, 2002). Hence, the emergence of the mother-inlaw in the puerperium as the peiyue kin can be double-edged.
Thus far, the epidemiology research has overlooked the role of conflict between mother-in-law/daughter-in-law in causing postnatal depression. Besides, the hypothesis that specific cultural practices can preclude postnatal depression - which can have profound public health implications - has not been subjected to empirical testing. These omissions are but examples that reveal the divide between ethnographic and epidemiological research. Ethnographic hypotheses are rarely tested empirically, and epidemiological studies seldom draw on ethnographic insights. We want to show that this divide is unnecessary, and can be overcome.

\section{Assessment tools}

Most risk factors were ascertained by direct inquiry with the participants or examination of case records. Psychometric rating scales were used to assess the following risk factors: marital relationship (Index of Marital Satisfaction), perceived social support (Medical Outcome Study Social Support Survey) and antenatal life events (Social Readjustment Rating Scale).

The Index of Marital Satisfaction (IMS) is a 25 -item self-report scale designed to measure the degree of problem a spouse encounters in the marital relationship (Hudson, 1982). It has been shown to have excellent internal consistency, test-retest reliability and concurrent validity. A score of 31 or above is commonly used to define marital problems. The psychometric properties of the Chinese IMS are comparable to the original version, and the validation data are currently under review for publication.

The Social Readjustment Rating Scale (SRRS) is a self-administered scale that has been widely used to examine 43 significant life events in the near past (Holmes $\&$ Rahe, 1967). The Chinese version was developed for the Hong Kong context: items irrelevant to local context were removed, and life events of indigenous importance were added (Shek \& Mak, 1997). The Chinese SRRS contains both threatening and rewarding events, as well as major and minor life events.

The Medical Outcome Study Social Support Survey is a 20-item selfadministered questionnaire developed by the Rand and Medical Outcomes Study teams to measure social support; it has good reliability and validity (Sherbourne
\& Stewart, 1991). The Chinese version has been validated, showing satisfactory psychometric properties (Yu et al, 2002).

The peiyue ritual was assessed with a semi-structured interview that focused on the content, context and duration of the practice. The semi-structured interview, developed by the research team, also assessed the knowledge and practice of other traditional post-partum customs. The first part of the assessment enquires about the respondent's knowledge of the contents of post-partum customs, i.e. whether the respondents are aware of the different taboos and rituals. The second part asks about the actual practice of various rituals and taboos after the delivery. The duration of, and the degree of adherence to, different rituals were recorded. This section also examines where, by whom and for how long the peiyue was observed. The participation of the father in child care and household duties in the peiyue period was also assessed.

The mother-in-law relationship was assessed with a modified five-point Likert measure, as no specific scale has been developed to measure this dyadic relationship. Our pilot test showed that framing an appropriate statement for the in-law relationship was difficult. A statement such as 'I am on poor terms with my mother-in-law' often attracts defensive responses, whereas the reverse statement 'I get on well with my mother-in-law' tends to attract socially approved answers. Hence, instead of asking the respondents to rate the level of agreement or disagreement with a statement, we ask them to rate the relationship with the mother-in-law, using one of the following responses ( 5 very good, 4 good, 3 average, 2 poor, 1 very poor). The respondents replied with a number instead of the corresponding adjective which has more explicit meaning. We also enhanced the veracity of the report by putting the question near the end of the interview when there was generally better rapport. In debriefing the respondents of the pilot study, we found this modified Likert measure to be superior to the conventional agreement approach. The 'poor' and 'very poor' responses were able to capture nearly all participants who had significant or serious problems with their mothers-in-law. Hence, we combined these two responses into one category, comparing it with a second category comprising the 'very good', 'good' and 'average' responses, in the statistical analyses. 


\section{Assessment of depression}

Postnatal depression was assessed using the Edinburgh Postnatal Depression Scale (EPDS; Cox et al, 1987). Numerous studies have demonstrated the reliability and validity of the EPDS in identifying depression, and it has been widely applied in both research and clinical settings. We validated the Chinese version of the EPDS and found that its sensitivity $(0.82)$ and specificity (0.86) were comparable to the original scale. A lower cut-off score (9/10) was desirable for the Chinese population (Lee et al, 1998). Since the EPDS was originally designed to screen for-rather than to diagnose - postnatal depression, the EPDS high scorers are referred to as 'probable postnatal depression' hereafter.

We also used the EPDS to assess level of depression in the third trimester. We did not use this scale to define probable depression in the third trimester because our previous analysis had shown that antenatal depression was rare in the study sample (Lee et al, 2001). Based on informed clinical experience, we categorised the thirdtrimester EPDS scores into three levels: $0-9$, nil or insignificant depressive symptoms; 10-14, mild to moderate depressive symptoms; and $>14$, severe depressive symptoms. These groupings were decided before data were collected.

\section{Data analyses}

The socio-demographic and psychosocial characteristics of the participants were summarised using descriptive statistics. The characteristics of the participants who were not included in the 3-month assessment were compared with those who were assessed using the chi-squared test for categorical data and the $t$-test for ordinal data Because nearly 20 hypotheses were tested in checking for potential biases introduced by 3-month attrition, a conservative threshold for significance $(P<0.01)$ was used instead of the conventional $P<0.05$.

The 3-month probable postnatal depression status was used as the dependent variable for univariate and multivariate analyses against putative risk factors. The risk of 'caseness' according to the presence or absence of risk factors was first assessed in a univariate manner, and risk ratios calculated with $95 \%$ confidence intervals. Statistical associations between caseness and individual putative risk factors were tested using $\chi^{2}$ tests for dichotomous and categorical variables, and independent Student's $t$-test for continuous variables. Because more than 30 hypotheses were tested in the univariate analyses, a conservative threshold for significance $(P<0.01)$ was used instead of the conventional $P<0.05$.

We entered factors that yielded risk ratios greater than 2 or confidence intervals that did not cross 1 on univariate analysis into logistic regression. Stepwise logistic regression analysis was first used to identify independent risk factors. When the best model was achieved, the regression was repeated using backward modelling to test for model stability. We computed unadjusted odds ratios and confidence intervals for each significant variable in the final model by the logistic regression. The adjusted odds ratios were computed by including all significant variables in the logistic regression.

\section{RESULTS}

\section{Sample characteristics}

A total of 1600 women were approached for recruitment and $959(60 \%)$ women participated. A total of 781 women $(81 \%)$ participated in the 3-month post-partum assessment and their socio-demographic characteristics are summarised in Table 1 . Of the 178 women $(19 \%)$ who left the study, 99 withdrew their consent, 56 could not be contacted and 23 had suffered a miscarriage. The mean age of the participants was 29.0 (s.d.=4.9) years and the mean number of children before the index pregnancy was 0.6 (s.d. $=0.8$ ). The participants who were not included in the 3-month follow-up were not different from those who remained in the study in terms of socio-demographic characteristics, past depressive episode and deliberate self-harm (Table 1). Baseline levels of depression, marital satisfaction and social support were not different between the two groups. However, those who left the study were more likely to be at a later stage of gestation when recruited $\left(\chi^{2}=35.46\right.$, d.f. $=2$, $P<0.001$ ).

\section{Peiyue support custom}

Among the women included in the 3-month post-partum assessment, 626 participants $(80.2 \%)$ received peiyue support in the first post-partum month, most commonly from their mothers-in-law $(35.3 \% ; n=221)$, their own mothers $(34.3 \% ; n=215)$ or other relatives $(26.8 \% ; n=168)$. Women who received peiyue care reported better social support than those who did not: $t=0.49$, d.f. $=202.6, P=0.013$; mean difference 7.8 $(95 \%$ CI $4.5-11.0)$, standard error of difference 1.7 .

\section{Univariate analysis}

A total of six putative risk factors were found to be significant in the univariate analysis (Tables 2 and 3). Past depression, marital dissatisfaction, inadequate social support, life events in pregnancy, depressive symptoms in the third trimester and poor mother-in-law relationship were associated with probable postnatal depression.

\section{Logistic regression analysis}

The six significant variables were entered into the stepwise logistic regression analyses. Because of theoretical interest, we also include peiyue in the regression analyses. The final model had four explanatory variables (Table 4). Backward stepwise regressions yielded the same model. The final model indicated that probable postnatal depression was predicted by past depression, depressive symptoms in late pregnancy, marital dissatisfaction and poor mother-in-law relationship. A dose effect was also observed for antenatal depressive symptoms.

\section{DISCUSSION}

Our study showed that the risk factors for postnatal depression established in Western societies are also applicable to a Chinese population. Among our participants, marital dissatisfaction, antenatal life events, past depression, late antenatal depressive symptoms and lack of social support predicted postnatal affective morbidity. Similar patterns of risk factors have been reported in other non-Western societies, including India, Taiwan, South Africa, Turkey and Dubai (Mills et al, 1995; Abou-Saleh \& Ghubash, 1997; Chandran et al, 2002; Inandi et al, 2002). It thus appears that there is substantial universality in the causation of postnatal depression across cultures.

\section{In-law dynamics in contemporary Asian families}

Local cultural factors are also pertinent in shaping maternal socio-affective wellbeing. In-law conflict was as important as spousal discord in determining postnatal emotional adjustment in our population; this confirms our ethnographic observation, and echoes the findings of other researchers 


\begin{tabular}{|c|c|c|c|c|c|}
\hline & $\begin{array}{l}\text { Women assessed at } 3 \text { months } \\
\text { post-partum }(n=78 \mathrm{I}) \\
n(\%)\end{array}$ & $\begin{array}{l}\text { Women not assessed at } 3 \text { months } \\
\text { post-partum }(n=178) \\
n(\%)\end{array}$ & $\chi^{2}$ & d.f. & $P$ \\
\hline Place of birth & & & 0.009 & $\mathrm{I}$ & 0.98 \\
\hline Hong Kong & $530(67.9)$ & $|2|(68.0)$ & & & \\
\hline Elsewhere & $251(32.1)$ & $57(32.0)$ & & & \\
\hline Martial status & & & 5.36 & $\mathrm{I}$ & 0.02 \\
\hline Married & 751 (96.2) & $164(92.1)$ & & & \\
\hline Not married & $30(3.8)$ & $14(7.9)$ & & & \\
\hline Education & & & 5.36 & 4 & 0.25 \\
\hline$<6$ years & II (I.4) & I (0.6) & & & \\
\hline 6 years & $96(12.4)$ & $15(8.4)$ & & & \\
\hline 9 years & $20 \mathrm{I}(25.7)$ & $40(22.5)$ & & & \\
\hline 12 years & $404(51.7)$ & $10 \mathrm{I}(56.7)$ & & & \\
\hline Tertiary & $69(8.8)$ & $21(11.8)$ & & & \\
\hline Occupation & & & 0.51 & 3 & 0.92 \\
\hline Unskilled/semi-skilled & $9(1 . I)$ & I (0.6) & & & \\
\hline Skilled & $366(46.9)$ & $83(46.6)$ & & & \\
\hline Semi-professional/professional & $52(6.7)$ & $12(6.8)$ & & & \\
\hline Home-maker & $354(45.3)$ & $82(46.1)$ & & & \\
\hline Social class & & & 6.35 & 4 & 0.17 \\
\hline $\mathrm{I}$ & $5(0.6)$ & I (0.6) & & & \\
\hline II & $149(19.1)$ & $48(27.0)$ & & & \\
\hline III & $587(75.2)$ & $|2|(68.0)$ & & & \\
\hline IV & 13 (1.7) & $4(2.2)$ & & & \\
\hline V & $27(3.5)$ & $4(2.2)$ & & & \\
\hline Gestation at recruitment (trimester) & & & 35.46 & 2 & $<0.00 \mathrm{I}$ \\
\hline First & $126(16.1)$ & 35 (19.7) & & & \\
\hline Second & $615(78.7)$ & $112(62.9)$ & & & \\
\hline Third & $40(5.1)$ & 31 (I7.4) & & & \\
\hline \multicolumn{6}{|l|}{ Psychiatric history } \\
\hline Depressive episode(s) & $53(6.8)$ & $15(8.4)$ & 0.59 & $\mathbf{I}$ & 0.42 \\
\hline Deliberate self-harm & $23(2.9)$ & 3 (I.7) & 0.87 & 1 & 0.45 \\
\hline Family history of mental disorder & $45(5.8)$ & $8(4.5)$ & 0.45 & I & 0.59 \\
\hline
\end{tabular}

(Barrows \& Barrows, 2002; Chan et al, 2002; Chandran et al, 2002). Adverse in-law relationship, especially between mother- and daughter-in-law, is a centuries-old conflict in Asian societies. For instance, a high percentage of Chinese couples considered the in-law relationships to be difficult (Tseng \& Hus, 1991). A Chinese national survey showed that trouble with the mother-in-law was the third most common stressful life event among married women (Zheng \& Lin, 1994). In another study in rural China, mother-in-law conflict was reported in nearly a third of young women who attempted suicide (Veronica, 2002). In Hong Kong and India, mothers-in-law play a significant role in domestic violence inflicted on married women (Fernandez, 1997; Leung et al, 2002). The mother-inlaw relationship is also a common area of conflict for urban Japanese housewives (Ago et al, 1982). In Korea, nearly a third of abused married women ascribed the problem to in-law troubles (Kim \& Shim, 1992).

The modern relationship between daughter-in-law and mother-in-law is embedded as much in the traditional patrilineal culture as in the growing economic independence of younger generations of Asian women. Traditionally in Asian societies, marriage means bringing in a daughter-in-law to join the family rather than composing a new household for the newly-weds. The daughter-in-law was commonly entrusted to the supervision and control of her mother-in-law, who was generally portrayed as tyrannical, if not abusive. The stereotypical representation of an oppressive mother-in-law and a submissive daughter-in-law, however, is rarely seen these days, as improved education opportunities and economic independence among younger women have remade the in-law relationships. These relationships are increasingly egalitarian, and family power has progressively been shifted to the younger generation (Rosen, 1978). This shift in power, however, does not necessarily reduce the tension imbued in the in-law relationship. The dissolution of archetypal hierarchy means that contemporary women now have to contest and 
Table 2 Risk factors significant by $\chi^{2}$-test for postnatal depression

\begin{tabular}{|c|c|c|c|c|c|c|}
\hline & $\begin{array}{l}\text { Postnatal depression } \\
\qquad \begin{array}{c}(n=122) \\
n\end{array}\end{array}$ & $\begin{array}{l}\text { No depression } \\
\qquad \begin{array}{c}(n=659) \\
n\end{array}\end{array}$ & $\chi^{2}$ & d.f. & Relative risk $(95 \% \mathrm{Cl})$ & $P$ \\
\hline \multicolumn{7}{|l|}{ Past depression } \\
\hline No & 105 & 623 & 11.7 & I & 1 & 0.001 \\
\hline Yes & 17 & 36 & & & $2.22(1.45-3.4 I)$ & \\
\hline \multicolumn{7}{|l|}{ Marital dissatisfaction } \\
\hline No & 48 & 396 & 26.5 & I & I & $<0.001$ \\
\hline Yes & 68 & 198 & & & $2.36(1.69-3.31)$ & \\
\hline \multicolumn{7}{|l|}{ Poor in-law relationship } \\
\hline No & 20 & 43 & 13.9 & I & 1 & $<0.00$ I \\
\hline Yes & 82 & 511 & & & $2.29(1.52-3.47)$ & \\
\hline \multicolumn{7}{|l|}{ Peiyue care } \\
\hline Yes & 90 & 536 & 4.0 & I & I & 0.046 \\
\hline No & 32 & $|2|$ & & & 1.45 (1.0 I-2.09) & \\
\hline \multicolumn{7}{|c|}{ Third trimester depressive symptoms } \\
\hline Nil or insignificant & 51 & 497 & 76.1 & 2 & 1 & $<0.001$ \\
\hline Mild to moderate & 35 & 92 & & & $2.96(2.02-4.35)$ & \\
\hline Severe & 27 & 29 & & & $5.18(3.55-7.55)$ & \\
\hline
\end{tabular}

negotiate for a new share of power. This negotiation is by no means easy: motherin-law and daughter-in-law, brought up in different value systems, often have diametrically opposite views on the interpersonal issues of autonomy, respect, hierarchy and filial piety.

It is thus unsurprising that the intragender and intergeneration conflicts between daughters-in-law and mothers-inlaw are difficult to deal with in the clinical context. The depressed daughters-in-law and their families may regard the conflicts as familial secrets that should not be disclosed to outsiders. Even when the daughters-in-law are willing to discuss the conflicts with therapists, most mothers-inlaw would not come for assessment, let alone Western-style psychotherapy. So far, Asian psychiatry has treated in-law conflict as just one of many possible forms of interpersonal difficulties. As such, treatment (when provided) is usually eclectic and non-specific. In order for interventions to be culturally specific and appropriate, more research is clearly needed.

\section{Rethinking maternal support and post-partum customs}

We found that the absence of the postpartum support custom (peiyue) was marginally significant $(P<0.05)$ in the univariate analysis, but not in the logistic regression. Given that more than 30 hypotheses were tested in the univariate analysis, it can be speculated that the significant association of peiyue was a spurious one. If a more conservative threshold of significance $(P<0.01)$ were used, the association would no longer be significant. On the other hand, a negative finding for peiyue in the logistic regression should not be unexpected, given that social support - a major ingredient of peiyue was adjusted for in the multivariate analysis. Furthermore, our study showed that

Table 3 Risk factors significant by Student's t-test for postnatal depression

\begin{tabular}{lccccc}
\hline & $\begin{array}{c}\text { Postnatal } \\
\text { depression } \\
(n=122) \\
\text { Mean (s.d.) }\end{array}$ & $\begin{array}{c}\text { No depression } \\
(n=659) \\
\text { Mean (s.d.) }\end{array}$ & $t$ & d.f. & $P$ \\
\hline Antenatal life events & $16.3(14.7)$ & $10.2(10.0)$ & 5.7 & 777 & $<0.00$ I \\
Social support & $70.8(18.0)$ & $79.5(16.5)$ & -5.3 & 779 & $<0.00$ I \\
\hline
\end{tabular}

Table 4 Risk factors selected by stepwise logistic regression for postnatal depression

\begin{tabular}{|c|c|c|c|}
\hline Risk factor & $\begin{array}{l}\text { Unadjusted odds ratio } \\
\qquad(95 \% \mathrm{Cl})\end{array}$ & $\begin{array}{l}\text { Adjusted odds ratio } \\
\qquad(95 \% \mathrm{Cl})\end{array}$ & $P$ \\
\hline \multicolumn{4}{|c|}{ Third trimester depressive symptoms } \\
\hline Nil or insignificant & I & I & \\
\hline Mild or moderate & $3.71(2.28-6.02)$ & $3.71(2.13-6.47)$ & $<0.001$ \\
\hline Severe & $9.07(4.99-16.50)$ & $5.66(2.70-11.88)$ & $<0.001$ \\
\hline Poor in-law relationship & $2.90(1.62-5.17)$ & $2.43(1.22-4.84)$ & 0.01 \\
\hline Marital dissatisfaction & $2.83(1.89-4.26)$ & $1.93(1.16-3.20)$ & 0.01 \\
\hline Past history of depression & $2.80(1.5 I-5.17)$ & $2.70(1.25-5.80)$ & 0.01 \\
\hline
\end{tabular}


the mother-in-law was a common person to provide peiyue. If the new mother and her mother-in-law did not get along with each other, the in-law tension or conflict could offset the potential benefit of the peiyue arrangement (Steinberg, 1996). Thus, mandated post-partum support, although wellintentioned, can be a mixed blessing; and this may explain why in this study peiyue is associated with only a slightly lower risk of depression. Future study should examine how the new mothers perceive the peiyue relationship, and differentiate between the helpful and unhelpful peiyue support arrangements in the investigation.

\section{Methodological issues}

Our study has several limitations that deserve mentioning. First, the diagnosis of postnatal depression was established with a self-report rating scale rather than a clinician-administered structured diagnostic interview. The EPDS was originally designed as a screening test, not a method for diagnosing postnatal depression; thus, although it has been increasingly used in large-scale postnatal depression studies to avoid burdening staff with timeconsuming diagnostic interviews (Warner et al, 1996), it is important to appreciate that its findings cannot be equated with psychiatric diagnoses. The EPDS can at best be used as an estimate of probable caseness; thus, participants with high scores on this scale received a group label of 'probable postnatal depression' in this report. Second, almost a fifth of the participants failed to attend 3-month assessment. Although these participants were essentially no different from those who completed the study in terms of socio-demographic or psychiatric characteristics, they were more likely to be later in their pregnancy at recruitment. Third, about $40 \%$ of eligible women declined to participate in the study, and only limited socio-demographic data were available from these non-participants to evaluate the potential bias they might have introduced. Furthermore, although we made the best effort to measure the risk factors before the onset of depression, some factors could only be evaluated at the 3month post-partum assessment. Besides, it is possible that 'post-partum' depression might actually begin as sub-syndromal depression or even as depressive symptoms in pregnancy. Hence, in some cases the risk factor assessment may not be entirely in advance of the outcome.
It is also important to note that our reported findings are associations rather than causations. The relationship between inlaw conflicts and postnatal depression can be more complicated than is portrayed in our statistical model. Apart from exerting a direct effect on maternal post-partum emotional well-being, the mother-in-law conflict may also interact with the new mother's personality or affective state. Women with certain personality traits may be more susceptible to mother-in-law conflicts. Likewise, women who have developed substantial depressive symptoms during pregnancy may also be more sensitive to in-law conflicts. It is also probable that personality traits or antenatal depressive mood independently contribute to the postnatal depression. To unravel the complex relationship, more studies are needed.

The study did not consider whether the mother-in-law can also suffer from conflict with her daughter-in-law. As it has been shown that maternal and paternal postnatal depression are closely connected, future study should examine if mothers-in-law can develop depression in response to the stresses of grandparenthood in this cultural setting. Finally, the study was conducted in one particular Chinese society. Replications in other Chinese and other Asian societies are warranted.

\section{Clinical implications}

A recent study in east London showed that the in-law relationship remains a powerful force in the local world of South Asian mothers living in the UK (Bhopal, 1998). Since the in-law relationship is essentially a peripheral one in Western family culture, clinicians may not be aware that the extended family is the main form of family life, and the in-laws are usually defined inside the family boundary of Asian families (Chen, 1999). Psychiatrists and general practitioners should therefore routinely consider the relevance of the motherin-law and other elder female kin when working with Asian families.

We hope this study will stimulate more research into the organisation of social support in the contemporary postpartum context. The myth that postpartum rituals and customs have largely disappeared in the industrialised world deserves examination. In many Western societies it is not uncommon for recently delivered women to be assisted by extended family members, some of whom may live in for a short period. More privileged families may even pay for a maternity nurse to support the new mother in the initial postpartum weeks (Morgan, 1996). A recent study in the UK reported that $41 \%$ of women practised some form of dietary proscription after childbirth (Huang \& Mathers, 2001). Yet, in contrast to peiyue and similar rituals, post-partum customs in Western societies are generally unnamed, unstructured and unnoticed. More research is thus needed to fathom the submerged post-partum customs of the industrialised world. Understanding how practical, emotional and informational support of new mothers is structured in contemporary society can have profound public health implications, as it may lead to more effective interventions and prevention programmes.

Last but not least, our study shows that it is possible to combine rich ethnographic observation with rigorous epidemiological testing. The result is a multi-level pathway connecting cultural tradition, family institution, interpersonal experience and maternal emotional well-being. As our world becomes ever more globalised and multiethnic, psychocultural understandings of this kind will be of increasing relevance to Western psychiatric practice.

\section{ACKNOWLEDGEMENT}

The study was supported by grant number 621019 of the Hong Kong Health Services Research Fund.

\section{REFERENCES}

Abou-Saleh, M.T. \& Ghubash, R. (1997) The

prevalence of early postpartum psychiatric morbidity in Dubai: a transcultural perspective. Acta Psychiatrica Scandinavica, 95, 428-432.

Ago, Y., Sugita, M., Teshima, H., et al (1982) Specificity concepts in Japan. Psychotherapy and Psychosomatics, 38, 64-73.

Appleby, L., Kumar, R. \& Warner, R. (1996) Perinatal psychiatry. International Review of Psychiatry, 8, 5-7.

Barrows, P. \& Barrows, K. (2002) Postnatal depression in Turkey: epidemiological and cultural aspects. Social Psychiatry and Psychiatric Epidemiology, 37, 125-129.

Becker, A. E. (1998) Postpartum illness in Fiji: a sociosomatic perspective. Psychosomatic Medicine, 60 43I-438.

Bhopal, K. (1998) South Asian women in East London: motherhood and social support. Women's Studies International Forum, 2I, 485-492.

Brugha, T. S., Sharp, H. M., Cooper, S. A., et al (1998) The Leicester 500 Project. Social support and the development of postnatal depressive symptoms, a prospective cohort survey. Psychological Medicine, 28 63-79. 
Chan, S. W., Levy, V., Chung, T. K., et al (2002) A qualitative study of the experiences of a group of Hong Kong Chinese women diagnosed with postnatal depression. Journal of Advanced Nursing, 39, 57I-579.

Chandran, M., Tharyan, P., Muliyil, J., et al (2002) Post-partum depression in a cohort of women from a rural area of Tamil Nadu, India: incidence and risk factors. British Journal of Psychiatry, 18I, 499-504.

Chen, M. H. (1999) The Relationship of Mothers and Daughters-in-Law in Urban Chinese Families (PhD dissertation). Denton, TX: Texas Woman's University.

Cox, J. L. (1996) Perinatal mental disorder - a cultura approach. International Review of Psychiatry, 8, 9-16.

Cox, J.L., Holden, J. M. \& Sagovsky, R. (1987)

Detection of postnatal depression: development of the 10-item Edinburgh Postnatal Depression Scale. British Journal of Psychiatry, 150, 782-786.

Fernandez, S. C. (1997) Domestic violence by extended family members in India: interplay of gender and generation. Journal of Interpersonal Violence, 12 456-465

Grace, J., Lee, K. K., Ballard, C., et al (200I) The relationship between post-natal depression, somatization and behaviour in Malaysian women Transcultural Psychiatry, 38, 27-34.

Holmes, T. H. \& Rahe, R. H. (1967) The Socia Readjustment Rating Scale. Journal of Psychosomatic Research, II, 213-218.

Huang, Y. C. \& Mathers, N. (200I) Postnata depression - biological or cultural? A comparative study of postnatal women in the UK and Taiwan. Journa of Advanced Nursing, 33, 279-287.

Hudson, W.W. (1982) The Clinical Measurement Package: A Field Manual. Chicago, IL: Dorsey.

Inandi, T., Elci, O. C., Ozturk, A., et al (2002) Risk factors for depression in postnatal first year, in eastern Turkey. International Journal of Epidemiology, 31, 1201-1207.

Kim, L. \& Shim, Y. (1992) A Study on the Prevalence and Policy Implications of the Domestic Violence in Seoul. Seoul: Korean Institute of Criminology.

Lee, D. T., Yip, S. K., Chiu, H. F., et al (1998) Detecting postnatal depression in Chinese women. Validation of the Chinese version of the Edinburgh Postnatal Depression Scale. British Journal of Psychiatry, I72, 433-437.

Lee, D. T., Yip, S. K., Chiu, H. F., et al (200I) A psychiatric epidemiological study of postpartum Chinese women. American Journal of Psychiatry, 158, 220-226.

Leung, W. C., Kung, F., Lam, J., et al (2002) Domestic violence and postnatal depression in a Chinese community. International Journal of Gynaecology and Obstetrics, 79, 159-166.

Mills, E. P., Finchilescu, G. \& Lea, S. J. (1995) Postnatal depression - an examination of psychosocial factors. South African Medical Journal, 85, 99-105.

Morgan, J. (1996) The new relationship with domestic staff. In Debrett's New Guide to Etiquette and Modern Manners, p. 297. New York: St Martin's Press.

O'Hara, K. W. \& Swain, A. M. (1996) Rates and risk of postpartum depression - meta-analysis. International Review of Psychiatry, 8, 37-54

Pillsbury, B. (1978) 'Doing the month': confinement and convalescence of Chinese women after childbirth. Socia Science and Medicine, $12,11-22$.

\section{CLINICAL IMPLICATIONS}

- The relationship between mother-in-law and daughter-in-law is as important as the marital relationship in shaping the emotional well-being of the daughter-in-law in Chinese society.

- Women who are continually supported by a designated female relative or friend in the first post-partum month reported better social support, and have a slightly lower risk of postnatal depression.

- In assessing families of Asian background, it is important to be aware that the extended family is the main form of family life, and the in-laws are commonly defined as being inside the family boundary.

\section{LIMITATIONS}

- About two-fifths of the eligible women did not participate in the study, and a fifth of the participants did not attend the post-partum assessment.

- The diagnosis of postnatal depression was established using the Edinburgh Postnatal Depression Scale rather than a diagnostic interview.

- The study was conducted in one Chinese society; replications in other Chinese and Asian societies are warranted.

DOMINIC T. S. LEE, MD, MRCPsych, Department of Social Medicine, Harvard Medical School, Boston Massachusetts, USA, and Department of Psychiatry, Chinese University of Hong Kong, Hong Kong; ALEXANDER S. K. YIP, MD, FRACOG, Department of Obstetrics and Gynaecology, Chinese University of Hong Kong; TONY Y. S. LEUNG, MPhil, Department of Psychiatry, Chinese University of Hong Kong; TONY K. H. CHUNG, MD FRACOG, Department of Obstetrics and Gynaecology, Chinese University of Hong Kong, Hong Kong

Correspondence: Dr DominicT. S. Lee, c/o Department of Psychiatry, Chinese University of Hong Kong, Shatin, Hong Kong. Fax: (617) 812 0077; e-mail: tak_lee@hms. harvard.edu

(First received 6 May 2003, final revision 27 August 2003, accepted 5 September 2003)

Rosen, S. (1978) Sibling and in-law relationships in Hong Kong. Journal of Marriage and the Family, 40, 62I-628.

Shek, D. T. L. \& Mak, J.W. K. (1997) Psychological Wellbeing of Working Parents in Hong Kong: Mental Health, Stress and Coping Responses. Hong Kong: Christian Service.

Sherbourne, C. D. \& Stewart, A. L. (1991) The MOS Social Support Survey. Social Science and Medicine, 32, 705-714.

Steinberg, S. (1996) Childbearing research: a transcultural review. Social Science and Medicine, $\mathbf{4 3}$ 1765-1784.

Stern, G. \& Kruckman, L. (1983) Multi-disciplinary perspectives on post-partum depression: an anthropological critique. Social Science and Medicine, I7 1027-1041.

Tseng, W. \& Hus, J. (1991) Culture and Family: Problems and Therapy. New York: Haworth Press.
Veronica, P. (2002) Attempted suicide among young rural women in the People's Republic of China: possibilities for prevention. Suicide and Life-Threatening Behavior, 32, 359-369.

Warner, R., Appleby, L., Whitton, A., et al (1996) Demographic and obstetric risk factors for postnatal psychiatric morbidity. British Journal of Psychiatry, 168, 607-611.

Yu, D. S. F., Lee, D. T. F. \& Woo, J. (2002) Establishing the Validity and Reliability of the Chinese Versions of the Chronic Heart Failure Questionnaire and the Medical Outcome Study Social Support Survey. Health Services Research Committee Report SII2002. Hong Kong: Health Services Research Fund.

Zheng, Y. P. \& Lin, K. M. (1994) A nationwide study of stressful life events in Mainland China. Psychosomatic Medicine, 56, 296-305. 